\title{
The MICE PID Instrumentation
}

\section{M.BONESINI ${ }^{* *}$}

Sezione INFN Milano Bicocca

E-mail: maurizio.bonesini@mib.infn.it

The international Muon Ionization Cooling Experiment (MICE) will carry out a systematic investigation of ionization cooling of a muon beam. As the emittance measurement will be done on a particle-by-particle basis, sophisticated beam instrumentation is needed to measure particle coordinates and timing vs RF. A PID system based on three time-of-flight stations, two Aerogel Cerenkov detectors and a KLOE-like calorimeter has been constructed in order to keep beam contamination $(e, \pi)$ well below $1 \%$. The MICE time-of-flight system will measure timing with a resolution better than 70 ps per plane, in a harsh environment due to high particle rates, fringe magnetic fields and electron backgrounds from RF dark current.

10th International Workshop on Neutrino Factories, Super beams and Beta beams

June 30 - July 52008

Valencia, Spain

\footnotetext{
* Speaker.

†n behalf of the MICE Collaboration
} 


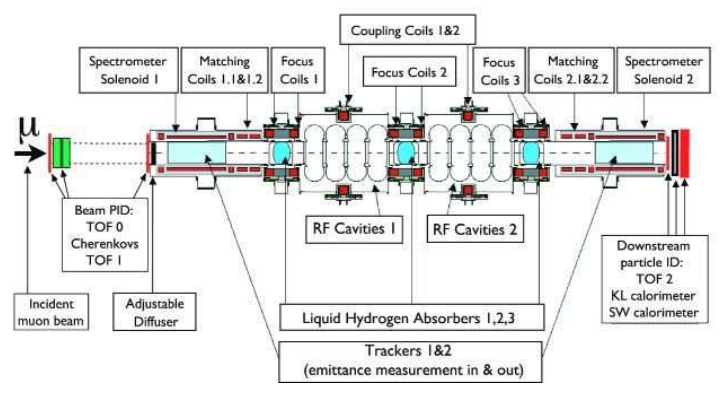

Figure 1: 2-D layout of the MICE experiment at RAL. The beam enters from the left. The cooling section is put between two magnetic spectrometers and two TOF stations (TOF1 and TOF2) to measure particle parameters.

\section{Introduction}

The neutrino factory $(v F)[1]$ is a muon storage ring with long straight sections, where decaying muons produce collimated neutrino beams of well defined composition and high intensity. Several $v \mathrm{~F}$ designs have been proposed, such as the ones of references [2,3]. A high intensity beam accelerated by a high power Proton Driver produces in a $\mathrm{Hg}$ jet target, after some accumulation and bunch compression, low energy pions. After a collection system, muons are cooled and phase rotated before acceleration up to $20 \mathrm{GeV} / \mathrm{c}$. Accelerated muons of well defined charge and momentum are then injected into an accumulator where they circulate until they decay, giving two neutrino beams along the straight sections. The physics program at a neutrino factory is very rich and includes long-baseline $v$ oscillations, short-baseline $v$ physics and slow muon physics [4]. The physics performance of a Neutrino Factory depends not only on its clean beam composition $\left(50 \% v_{e}, 50 \% \bar{v}_{\mu}\right.$ for the $\mu^{+} \mapsto \bar{v}_{\mu} v_{e} e^{+}$case), but also on the available beam intensity. The cooling of muons (accounting for $\sim 20 \%$ of the final costs of the factory) is thus compulsory, increasing the performance up to a factor 10. Due to the short muon lifetime $(2.2 \mu \mathrm{s})$, novel methods such as the ionization cooling, proposed more than 20 years ago by A.N. Skrinsky [5], must be used. Essentially the cooling of the transverse phase-space coordinates of a muon beam can be accomplished by passing it through an energy-absorbing material and an accelerating structure, both embedded within a focusing magnetic lattice. Both longitudinal and transverse momentum are lost in the absorber while the RF-cavities restore only the longitudinal component. The MICE experiment [6] at RAL aims at a systematic study of a section of a cooling channel (see figure 1 for a layout). A secondary muon beam from ISIS (140-240 MeV/c central momentum, tunable between 1-10 $\pi$. $\mathrm{mm}$ rad input emittance) enters a cooling section after a diffuser. The $5.5 \mathrm{~m}$ long cooling section consists of three absorbers and eight RF cavities encircled by lattice solenoids. The cooling process will be studied by varying the relevant parameters, to allow the extrapolation to different cooling channel designs.

\section{Emittance measurement}

Different $v \mathrm{~F}$ designs need a muon cooling factor from 2 to 16 , over a distance of $\sim 100 \mathrm{~m}$. 
The different designs of the cooling section have to be tested at least with a precision of $\sim 10 \%$. For an affordable cooling section prototype a cooling $\sim 5-20 \%$ may be exptected. This implies emittance measurements at a level of $0.1 \%$, thus excluding conventional emittance measurement methods, with precisions around $10 \%$. To obtain such a pecision a method based on single particle measurements has been envisaged.

Particles are measured before and after the cooling section by two magnetic spectrometers complemented by TOF detectors. For each particle $\mathrm{x}, \mathrm{y}, \mathrm{t}, \mathrm{x}^{\prime}=\mathrm{dx} / \mathrm{dz}=p_{x} / p_{z}, \mathrm{y}^{\prime}=\mathrm{dy} / \mathrm{dz}=p_{y} / p_{z}, \mathrm{t}^{\prime}=\mathrm{dt} / \mathrm{dz}$ $=E / p_{z}$ coordinates are measured. In this way, for an ensemble of $\mathrm{N}$ particles, the input and output emittances may be determined with high precision.

\section{The MICE PID system}

The driving design criteria of the MICE detector system are robustness, in particular of the tracking detectors, to sustain the severe background conditions in the vicinity of RF cavities and redundancy in PID in order to keep contaminations $(e, \pi)$ well below $1 \%$. Precision timing measurements are required to relate the time of a muon to the phase of the RF and simultaneously for particle identification by time-of-flight (TOF). A time resolution around $70 \mathrm{ps}\left(\sigma_{t}\right)$ provides both effective $(99 \%)$ rejection of beam pions and adequate $\left(5^{\circ}\right)$ precision of the RF phase. Particle identification (PID) is obtained upstream the first solenoid by two TOF stations (TOF0/TOF1) and two threshold Cerenkov counters (CKOVa/CKOVb), that will provide $\pi / \mu$ separation up to $300 \mathrm{MeV} / \mathrm{c}$. Downstream the PID is obtained via a further TOF station (TOF2) and a calorimeter (EMCAL), to separate muons from decay electrons.

Due to the large range of beam momenta, it is not possible to select a single material as Cerenkov radiator that is sensitive to muons and blind to pions over the entire range. The chosen solution is two different aerogel counters with refractive indices 1.07 and 1.12, each equipped respectively with four 8" low background EMI9356KA PMTs from the earlier Chooz experiment. Thus high purities are obtained: from $99.7 \%$ with both counters on to $99.98 \%$ with both counters off in the momentum range between 210 and $365 \mathrm{MeV} / \mathrm{c}$. At lower momenta, the $\pi / \mu$ separation is obtained by a tof measurement, both Cerenkov detectors being blind to both particle types.

All the TOF stations share a common design based on fast 1" scintillator counters along X/Y directions (to increase measurement redundancy) read at both edges by R4998 Hamamatsu photomultipliers ${ }^{1}$. While TOF0 planes cover a $40 \times 40 \mathrm{~cm}^{2}$ active area, TOF1 and TOF2 cover respectively a $42 \times 42 \mathrm{~cm}^{2}$ and $60 \times 60 \mathrm{~cm}^{2}$ active area. The counter width is $4 \mathrm{~cm}$ in TOF0 and $6 \mathrm{~cm}$ in the following ones. All downstream detectors and the TOF1 station must be shielded against stray magnetic fields (up to 1000-1500 Gauss with a $\leq 400$ Gauss longitudinal component, depending on the design of the shielding plates after the spectrometer solenoids). Two options for the local TOF1/TOF2 shielding are foreseen: in one (for TOF1) a double-sided shielding cage will contain fully the detector, aside a hole for the beam, while in the other (for TOF2) individual massive soft iron box PMTs shielding will be used [7]. While the first solution is more elegant and reduces the detector weight, it gives complications for detector access and maintenance. Figure 2 shows some results for the shielding of the most dangerous component of the B field, along the PMT axis,

\footnotetext{
${ }^{1} 1$ " linear focussed PMTs, typical gain $G \sim 5.7 \times 10^{6}$ at B=0 Gauss, risetime $0.7 \mathrm{~ns}$, TTS $\sim 160 \mathrm{ps}$, equipped with active/passive divider and a mu-metal shield extending $3 \mathrm{~cm}$ in front of the photocathode (H6533 assemblies)
} 

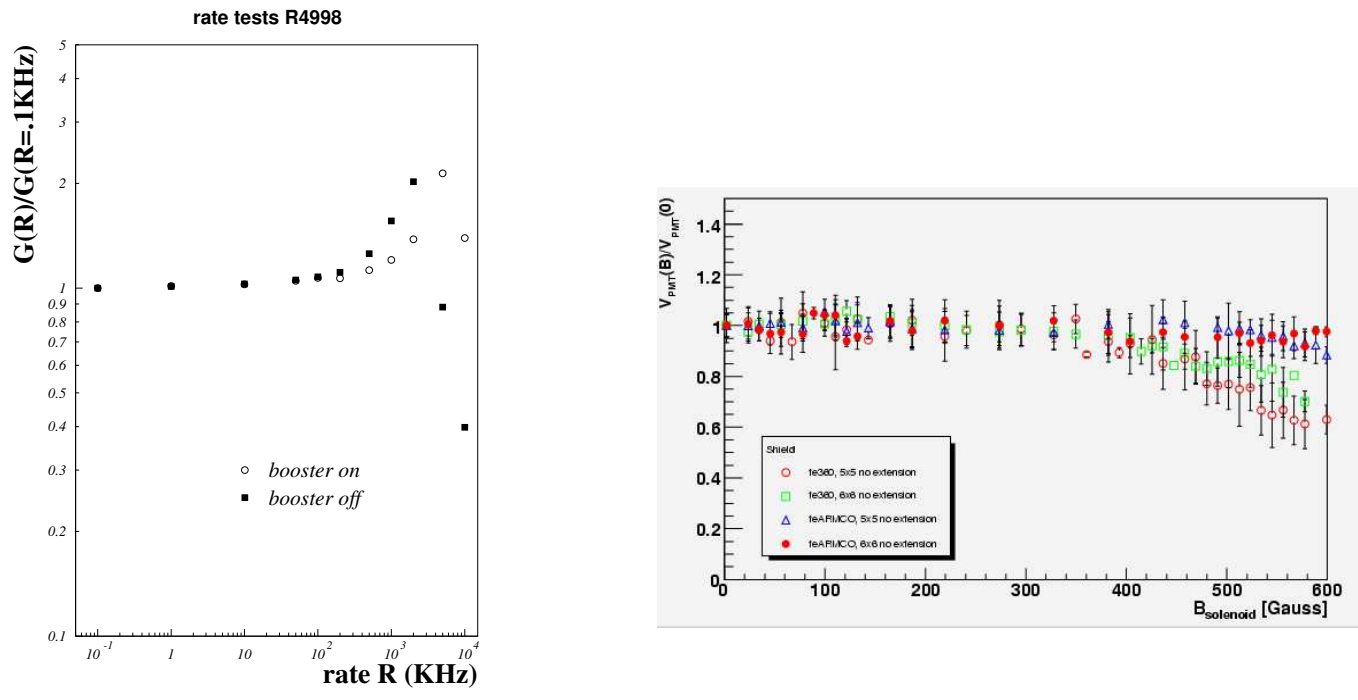

Figure 2: Left panel: rate capability of a typical R4998 PMT, as a function of rate R at field B=0 G (measured P.H. in $\mathrm{mV}$ versus rate in KHz). Right panel: tests of shieldings for conventional R4998 Hamamatsu PMTs. Results are shown for the average and rms of a sample of 10 PMTs, shielded with $5 \times 5$ or $6 \times 6 \mathrm{~cm}^{2}$ low carbon content (Fe360) (open circle or open square) or ARMCO (open triangle or black dot) soft iron box surrounding the mu-metal of the H6533 assemblies. The B field is along the PMT axis.

obtained with mu-metal+a massive iron box shielding. With ARMCO soft iron a good shielding is obtained up to $\sim 600 \mathrm{G}$.

The TOF stations must sustain a high instantaneous incoming particle rate (up to $1.5 \mathrm{MHz}$ for TOF0). PMT rate capabilities were tested in the laboratory with a dedicated setup [8] based on a fast laser. A typical R4998 PMT had a good rate capability for signals comparable to an incident $\mu$ ( $\sim 300$ p.e.) up to $\sim 1 \mathrm{MHz}$. The rate capability may be increased by the use of active bases or a booster on the last dynodes for the R4998 PMTs, as shown in figure 2.

Counter prototypes have been tested at the LNF DAФNE Beam Test Facility (BTF) with incident electrons of $E_{\text {beam }}=200-350 \mathrm{MeV}$ to study the intrinsic counter time resolution. The front end readout used the baseline MICE choice for the TDC: a multihit/multievent CAEN V1290 TDC, in addition to a CAEN V792 QADC (to be replaced in the experiment by CAEN V1724 FADC) for time-walk corrections. The PMT signal was split by a passive splitter followed by a leading-edge discriminator before the TDC line. An intrinsic single counter resolution $\sim 45-60 \mathrm{ps}$ was obtained depending upon beam conditions and the design of lightguides or the scintillator chosen (Bicron BC404 or BC420 ${ }^{2}$, Amcrys-H UPS95F). With a gaussian fit for the pulse-height distributions, the number of photoelectrons per single impinging electron $\left(N_{p e}\right)$ was estimated. From $\left(<R>/ \sigma_{R}\right)^{2}$, where $\left\langle R>\right.$ is the peak of the gaussian and $\sigma_{R}$ its width, an estimate in the range 200-300 p.e. for BC420 was obtained, depending on the impact point. Clearly, this estimation neglects electronic noise and is affected by the bad (good) scintillator-PMT coupling.

The downstream calorimeter (EMCAL) is a Pb-scintillating fiber calorimeter (KL), of the KLOE type [9], with 1-mm diameter blue scintillating fibers glued between $0.3 \mathrm{~mm}$ thick grooved lead plates followed by a muon ranger (SW), made of a $1 \mathrm{~m}^{3}$ fully sensitive segmented scintillator

\footnotetext{
${ }^{2}$ risetime $0.7(0.5) \mathrm{ns}, \lambda_{\text {emission }}^{\max }=408(391) \mathrm{nm}, \lambda_{\text {att }}^{\text {bulk }}=160(110) \mathrm{cm}$ for BC404 (BC420)
} 


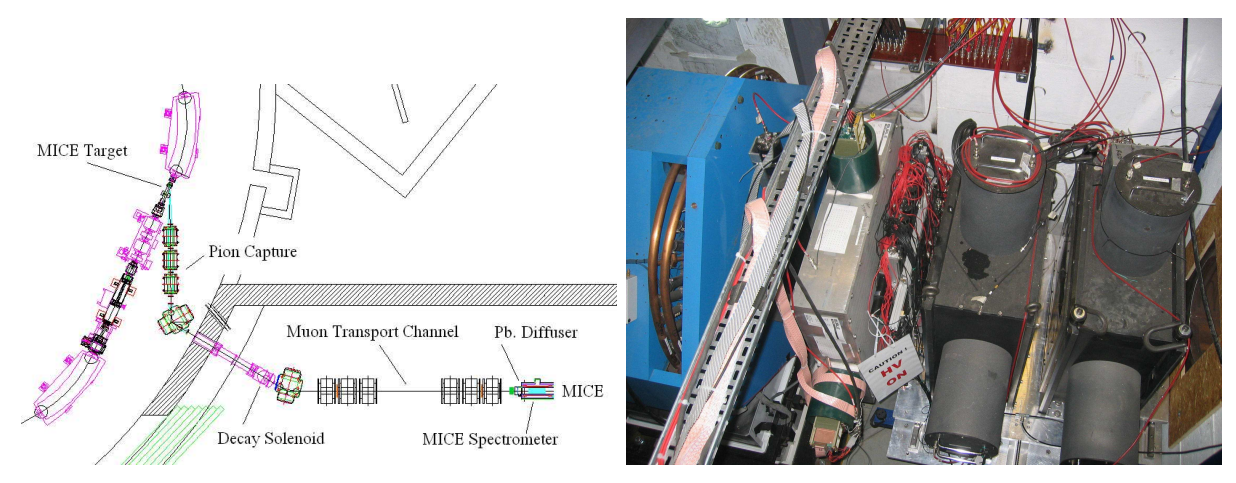

Figure 3: Left: layout of the MICE secondary beamline. $800 \mathrm{MeV} / \mathrm{c}$ protons from ISIS impinge on a Ti target. Produced pions are then guided to a decay solenoid, where muons are then steered to the MICE apparatus. The upstream detectors TOF0 and CKOVa/b are inserted between the two triplets of quads. Right: picture of the installed TOF0,CKOVa,CKOVb upstream detectors after the first quads triplet.

block. This "spaghetti" design for KL offers the possibility of a fine sampling and optimal lateral uniformity. The expected resolution $\sigma_{E} \simeq 5 \% / E$ is fully dominated by sampling fluctuations and is linear for electrons or photons in the range 70-300 MeV. SW will be made with extruded scintillator bars with WLS fibers readout. A prototype has been recently tested at CERN with good results. In the EMCAL while KL will measure electrons, $\mathrm{SW}$ will measure precisely the muon range.

\section{MICE installation and data taking}

MICE will be accomplished in steps, corresponding mainly to first characterizing the incoming beam and demonstrating the capability to do a high precision measure of emittance and then to measure the cooling for a variety of experimental situations. Up to now, only the first phase is under way: the beamline has been completed and the upstream PID detectors and KL installed, see figure 3 .

\section{References}

[1] D.G. Kosharev, CERN/ISR-DI/74-62 (174).

[2] M.M. Alsharo'a et al., Phys. ReV. ST. Accel. Beams 6,081001 (2003).

[3] A. Blondel et al., CERN-2004-002.

[4] M. Bonesini, A.Guglielmi Phys. Rep. 433 (2006) 65.

[5] A.N. Skrinsky, V.V. Parkhomchuk Sov. Jour. Nucl. Phys. 12 (1981) 3.

[6] A. Blondel et al., MICE proposal, RAL, 2004; G. Gregoire et al., MICE Technical Report, RAL, 2005.

[7] R. Stephens et al., D0 note 2706, 1996.

[8] M. Bonesini et al., Nucl. Instr. and Meth. A 572 (2007) 465,

M. Bonesini et al., Nucl. Instr. and Meth. a 567 (2006) 200.

[9] A. Aloisio et al., KLOE coll., Nucl. Inst. and Meth. A 494 (2002), 326. 